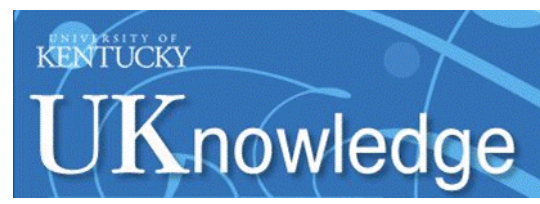

University of Kentucky

UKnowledge

4-10-1997

\title{
Accurate Hydrogen Spectral Simulations with a Compact Model
}

\section{Atom}

Jason W. Ferguson

University of Kentucky

Gary J. Ferland

University of Kentucky, gary@uky.edu

Follow this and additional works at: https://uknowledge.uky.edu/physastron_facpub

Part of the Astrophysics and Astronomy Commons, and the Physics Commons

Right click to open a feedback form in a new tab to let us know how this document benefits you.

\section{Repository Citation}

Ferguson, Jason W. and Ferland, Gary J., "Accurate Hydrogen Spectral Simulations with a Compact Model Atom" (1997). Physics and Astronomy Faculty Publications. 123.

https://uknowledge.uky.edu/physastron_facpub/123

This Article is brought to you for free and open access by the Physics and Astronomy at UKnowledge. It has been accepted for inclusion in Physics and Astronomy Faculty Publications by an authorized administrator of UKnowledge. For more information, please contact UKnowledge@lsv.uky.edu. 


\section{Accurate Hydrogen Spectral Simulations with a Compact Model Atom}

Digital Object Identifier (DOI)

http://dx.doi.org/10.1086/303880

\section{Notes/Citation Information}

Published in The Astrophysical Journal, v. 479, no. 1, p. 363-370.

(C) 1997. The American Astronomical Society. All rights reserved. Printed in the U.S.A.

The copyright holder has granted permission for posting the article here. 
The Astrophysical JouRnal, 479:363-370, 1997 April 10

(C) 1997. The American Astronomical Society. All rights reserved. Printed in U.S.A.

\title{
ACCURATE HYDROGEN SPECTRAL SIMULATIONS WITH A COMPACT MODEL ATOM
}

\author{
Jason W. Ferguson and Gary J. FerLand \\ Department of Physics and Astronomy, University of Kentucky, Lexington, KY 40506 \\ Received 1996 Seeptember 9 ; accepted 1996 October 23
}

\begin{abstract}
Many large-scale numerical simulations of astrophysical plasmas must also reproduce the hydrogen ionization and the resulting emission spectrum, in some cases quite accurately. We describe a compact model hydrogen atom that can be readily incorporated into such simulations. It reproduces the recombination efficiency and line spectrum predicted by much larger calculations for a very broad range of densities and temperatures. Uncertainties in hydrogen collision data are the largest source of differences between our compact atom and predictions of more extensive calculations and underscore the need for accurate atomic data.

Subject headings: atomic processes — plasmas
\end{abstract}

\section{INTRODUCTION}

Hydrogen is the most abundant element, and its physics dominates the conditions in a wide variety of galactic and extragalactic nebulae (Osterbrock 1989). Hydrogen's recombination efficiency and level populations must be computed with some precision, since its photoionization can be the controlling heating mechanism for many clouds, it is the dominant opacity for many forms of light, and $\mathrm{H}$ is often the dominant electron donor (Avrett \& Loeser 1988; Rees, Netzer, \& Ferland 1989; Netzer 1990). The line spectrum must be predicted with great precision if reliable abundance determinations are to be achieved (Martin 1988; Shields 1990; Pagel 1992). Modern spectral synthesis codes (see Kallman \& Mushotzky 1985; Binette et al. 1993; Ferland et al. 1995; Netzer 1996) must incorporate complete simulations of the $\mathrm{H}$ atom if they are to be valid.

A number of physical processes affects hydrogen, including radiative and three-body recombination, photoionization and induced recombination, and collisional and radiative (spontaneous and induced) transitions between levels. Complete calculations of the physics of the hydrogen atom are intricate themselves (Brocklehurst 1971; Mathews, Blumenthal, \& Grandi 1980; Drake \& Ulrich 1980; Storey \& Hummer 1995), and certainly cannot be incorporated into larger structure and spectral synthesis codes. These processes are important in the broad-line regions (BLRs) of quasars or any dense gas and so must be fully simulated.

Storey \& Hummer (1995, hereafter SH), have completed full calculations of the hydrogen emission spectrum and recombination efficiency over a very broad range of temperature and density. Their calculations are for "case A" and "case B" conditions, with the 1000 lowest $n$ levels and all $l$ states included. These computations are likely to remain definitive for some time to come, and they made their results available electronically, along with a convenient interpolating program. Unfortunately, it is possible to incorporate model hydrogen atoms with only dozens, not hundreds or thousands, of levels into full spectral synthesis simulations. The problem is to define a compact model atom which retains the essential physics and agrees with the definitive calculations.

This paper outlines a model atom that can accurately reproduce the recombination efficiency and line emission with only a few dozen quantum levels and can be incorpor- ated into large-scale structure simulations. The approach goes to the correct asymptotic limits and reproduces accurate results in intermediate conditions. We pay particular attention to obtaining accurate representations of emissivities of infrared lines. Surprisingly, the rates for collisions within the hydrogen atom appear to be the limiting factor on accuracy. Finally, we compare the idealized case B emissivity with results from a realistic BLR cloud model.

\section{PHYSICAL PROCESSES AND THEIR SIMULATION WITH A COMPACT ATOM}

Our model hydrogen atom consists of independent $1 s, 2 s$, and $2 p$ states and an arbitrary number of higher excited $n$-levels.

Both the low-density and LTE limits, as well as the intermediate "case A" and "case B" are well defined in the literature. Case B comes closest to simulating nature, and we concentrate on it here. For this approximation, all transitions involving the ground state are ignored, as is photoionization from excited states, collisional ionization and excitation from the ground and first excited states, fluorescent excitation, and all induced processes. These are normally included in our calculations but are disabled in the work presented below.

\subsection{Photoionization, Radiative Recombination}

Recombination to an infinite sum of bound levels must be included if the total recombination coefficient, and resulting ionization balance, are to be correct.

We have fitted radiative recombination coefficients for levels up to 15 using the Milne relation and photoionization cross sections taken from opacity project-related codes, as described in Ferland et al. (1992). The fit has the functional form

$$
\alpha(n, T)=10^{F(n, T)} T_{e}^{-1},
$$

where

$$
F\left(n, T_{e}\right)=\frac{a_{n}+c_{n} x+e_{n} x^{2}+g_{n} x^{3}+i_{n} x^{4}}{1+b_{n} x+d_{n} x^{2}+f_{n} x^{3}+h_{n} x^{4}}
$$

and $x=\log T_{e}$. Table 1 gives the coefficients used for the above equation, which are valid for the temperature range $2.8 \mathrm{~K} \leq T_{e} \leq 10^{10} \mathrm{~K}$. 
TABLE 1

COEFFICIENTS FOR EQUATION (1)

\begin{tabular}{|c|c|c|c|c|c|c|c|c|}
\hline \multirow[b]{2}{*}{ COEFFICIENT } & \multicolumn{8}{|c|}{$n$} \\
\hline & 1 & 2 & 3 & 4 & 5 & 6 & 7 & 8 \\
\hline$a \ldots \ldots \ldots$ & -10.78145 & -11.04340 & -11.20313 & -11.31452 & -11.40622 & -11.48461 & -11.46468 & -11.50326 \\
\hline$b \ldots \ldots \ldots \ldots$ & -0.38890 & -0.39351 & -0.42458 & -0.43939 & -0.43647 & -0.44246 & 0.03271 & -0.17837 \\
\hline$c \ldots \ldots \ldots$ & 4.68469 & 4.84334 & 5.25370 & 5.45398 & 5.46028 & 5.56951 & -0.07602 & 2.30302 \\
\hline$d \ldots \ldots \ldots \ldots$ & 0.06404 & 0.06921 & 0.08192 & 0.08538 & 0.08639 & 0.08998 & 0.00660 & 0.05393 \\
\hline$e \ldots \ldots \ldots \ldots$ & -0.87423 & -0.95686 & -1.12613 & -1.16660 & -1.18431 & -1.24103 & 0.02975 & -0.53187 \\
\hline$f \ldots \ldots \ldots \ldots$, & -0.00510 & -0.00550 & -0.00682 & -0.00740 & -0.00737 & -0.00755 & 0.01032 & -0.00120 \\
\hline$g \ldots \ldots \ldots$ & 0.08141 & 0.09216 & 0.11422 & 0.11806 & 0.11914 & 0.12544 & -0.10863 & 0.0 \\
\hline$h \ldots \ldots \ldots \ldots$ & 0.00248 & 0.00306 & 0.00412 & 0.00404 & 0.00412 & 0.00449 & -0.00253 & 0.0 \\
\hline$i \ldots \ldots \ldots \ldots$ & -0.03877 & -0.05025 & 0.0 & -0.06488 & -0.06752 & -0.07536 & 0.0 & 0.0 \\
\hline
\end{tabular}

\begin{tabular}{|c|c|c|c|c|c|c|c|c|}
\hline \multirow[b]{2}{*}{ COEFFICIENT } & \multicolumn{7}{|c|}{$n$} & \multirow[b]{2}{*}{ CASE B } \\
\hline & 9 & 10 & 11 & 12 & 13 & 14 & 15 & \\
\hline$a \ldots$ & -11.71000 & -11.61098 & -11.99276 & -11.71513 & -11.66252 & -11.69889 & -11.71537 & -9.97652 \\
\hline$b \ldots \ldots \ldots \ldots$ & 8.07670 & -0.16697 & 4.76460 & -0.14672 & 0.02722 & 0.02892 & 0.02312 & 0.03506 \\
\hline$c \ldots \ldots \ldots \ldots$ & -92.51728 & 2.20472 & -54.29910 & 2.02504 & -0.28688 & -0.28812 & -0.28765 & 0.15861 \\
\hline$d \ldots \ldots \ldots \ldots$ & -1.46915 & 0.05915 & -0.88171 & 0.06253 & 0.09368 & 0.09033 & 0.11802 & -0.03762 \\
\hline$e \ldots \ldots \ldots \ldots$ & 18.56208 & -0.59021 & 10.94495 & -0.63483 & -0.71041 & -0.69566 & -0.94380 & 0.30113 \\
\hline$f \ldots \ldots+\cdots$ & 0.51035 & -0.00135 & 0.33187 & -0.00146 & 0.00046 & 0.00173 & -0.00225 & 0.00762 \\
\hline$g \ldots \ldots \ldots \ldots$ & -4.93019 & 0.0 & -3.22476 & 0.0 & -0.05483 & -0.06497 & -0.03708 & -0.06397 \\
\hline h ............ & -0.12090 & 0.0 & -0.07931 & 0.0 & -0.00014 & -0.00048 & 0.00047 & -0.00023 \\
\hline$i \ldots \ldots \ldots \ldots$ & 0.0 & 0.0 & 0.0 & 0.0 & 0.0 & 0.0 & 0.0 & 0.00127 \\
\hline
\end{tabular}

For quantum levels above 15 we use an an asymptotic analytical formula from Allen (1973):

$$
\alpha_{n}=\left(3.262 \times 10^{-6}\right) n^{-3} T_{e}^{-3 / 2} \exp \left(\frac{\chi_{n}}{k T_{e}}\right) E_{1}\left(\frac{\chi_{n}}{k T_{e}}\right),
$$

where $\chi_{n}$ is the ionization potential of the $n$th level and $E_{1}$ is the first exponential integral (Abramowitz \& Stegun 1965).

Errors in individual recombination coefficient fits are generally $1 \%-2 \%$ with many less than $1 \%$ for lower excited states at temperatures of $10,000 \mathrm{~K}$. For levels $1 \leq n \leq 15$ the largest errors are $5 \%$ at only the highest temperatures. Equation (2), used for levels $n \geq 16$, breaks down at temperatures greater than $10^{8} \mathrm{~K}$, however at these high temperatures less that $2 \%$ of the total recombinations are to levels greater than 16 , so this does not introduce an error in the total recombination coefficient at these high temperatures.

The approach given above provides rates only for levels explicitly included in our model atom and thus is an underestimate of the total recombination coefficient, a sum to infinity. The total recombination coefficient must be accurately computed to produce accurate ionization representations in the low-density limit. To compensate, the highest five levels of the atom are assigned the remainder of the recombination coefficient:

$$
\alpha_{N}=\alpha_{\mathrm{B}}-\sum_{n=2}^{n=N-1} \alpha_{n},
$$

where $\alpha_{\mathrm{B}}$ is the total case B rate (the sum of recombinations to $n=2$ and higher levels fitted with eq. [1] and coefficients listed in Table 1), $N$ is the total number of levels in the compact model atom, and $\alpha_{n}$ is the recombination coefficient to the $n$th level. Although assigning the remaining coefficient to the higher levels had the desired effect of including all the possible recombinations, it also resulted in the overpopulation of these levels. This caused a level inversion and resulted in a strong maser and so was unphysical. We topped off the atom with a band of levels rather than the highest level to minimize this effect. The result of this topping off of the atom is that the model atom reproduces the $\alpha_{\mathrm{B}}$ sum and the ionization balance at low densities to very great precision.

\subsection{Collisional Ionization, Three-Body Recombination}

Collisional ionization and its reverse process, three-body recombination, are important since they bring the atom into LTE at high densities. Collisional ionization from the ground state is taken from Arnaud \& Rothenflug (1985). For levels 2 and higher we use rate coefficients of Vriens \& Smeets (1980), who give a semiempirical fit between theory and experiment for hydrogenic atoms. If the atom is in an initial excited state $|q\rangle$ with energy $E_{q}=13.6 \mathrm{eV} / q^{2}$, then the rate coefficient for ionization is

$$
K_{q}=\frac{9.56 \times 10^{-6}\left(k T_{e}\right)^{-1.5} \exp \left(-\varepsilon_{q}\right)}{\varepsilon_{q}^{2.33}+4.38 \varepsilon_{q}^{1.72}+1.32 \varepsilon_{q}},
$$

where $\varepsilon_{q}=E_{q} / k T_{e}$ is the Boltzmann factor with $k T_{e}$ in $\mathrm{eV}$, and the equation is good for the values of $k T_{e}$ given by Vriens \& Smeets (generally for temperatures greater than $10^{3} \mathrm{~K}$ ). For reference, the definitive $\mathrm{SH}$ calculations use collision data from Burgess \& Percival (1968). Chang, Avrett, \& Loeser (1991) discuss the uncertainties of the collision data for hydrogen. For intermediate levels this is easily a factor of 2 .

Figure 1 shows the total recombination coefficient for three temperatures and a wide range of density. The solid line presents the results with a 50-level atom, and the dashed line presents the SH results. At low densities the recombination coefficient is purely radiative, while threebody recombination dominates the total at high density. For low temperatures and intermediate densities the differences can be as great as $5 \%$. Tests discussed below show that the differences in our total recombination coefficient and those of SH are due to the different collision data assumed and so are a basic uncertainty. 


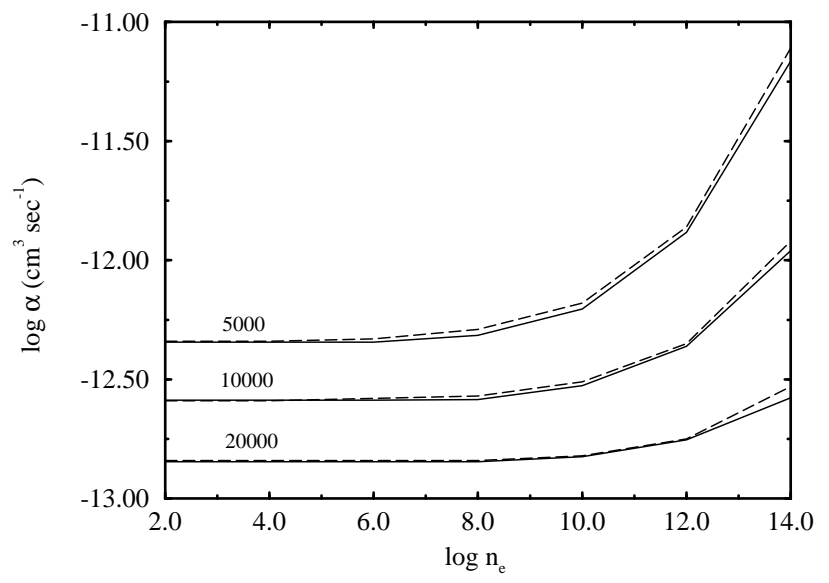

FIG. 1.-Total case B recombination coefficient defined as the radiative recombination plus three-body recombination minus collisional ionization, compared with the results of SH. Solid lines: this work; dashed lines: $\mathrm{SH}$. Three temperatures are shown, $5000 \mathrm{~K}, 10,000 \mathrm{~K}$, and 20,000 K. The differences are due to different sources of collisional ionization rate coefficients.

\section{3. $n$-Changing Collisions}

For collisional de-excitation involving the ground, first, and second excited states of hydrogen we use data from Callaway (1994); excitation rates are calculated by the principle of detailed balance. Collisions between $2 s$ and $2 p$ are taken from Osterbrock (1989). For collisional de-excitation involving levels 4 and higher we again use the formulae of Vriens \& Smeets (1980), which is available in closed form, is recommended by Chang, Avrett, \& Loeser (1991), and can be extended to any transition from any excited state. The atom is in an initial upper level $|q\rangle$ and de-excites to a lower level $|p\rangle$ with energy $E_{p}=13.6 \mathrm{eV} / p^{2}$ and energy difference $E_{p q}=13.6 \mathrm{eV}\left(1 / p^{2}-1 / q^{2}\right)$; then the de-excitation rate coefficient is given by

$$
\begin{aligned}
K_{q p}= & \frac{1.6 \times 10^{-7}\left(k T_{e}\right)^{0.5} g_{p} / g_{q}}{k T_{e}+\Gamma_{p q}} \\
& \times\left[A_{p q} \ln \left(\frac{0.3 k T_{e}}{\mathfrak{R}}+\Delta_{p q}\right)+B_{p q}\right],
\end{aligned}
$$

where $g_{p}=2 p^{2}$ and $g_{q}=2 q^{2}$ are the statistical weights. Defining $s=|p-q|$,

$$
\Delta_{p q}=\exp \left(\frac{-B_{p q}}{A_{p q}}\right)+\frac{0.06 s^{2}}{p q^{2}}
$$

and

$$
\begin{gathered}
\Gamma_{p q}=\frac{\mathfrak{R} \ln \left(1+q^{3} k T_{e} / \mathfrak{R}\right)\left[3+11(s / q)^{2}\right]}{\left(6+1.6 p s+0.3 / s^{2}+\left(0.8 p^{1.5} / s^{0.5}\right)|s-0.6|\right)} \\
A_{p q}=\left(\frac{2 \mathfrak{R}}{E_{p q}}\right) f_{p q}
\end{gathered}
$$

and

$$
B_{p q}=\frac{4 \Re^{2}}{p^{3}}\left(\frac{1}{E_{p q}^{2}}+\frac{4 E_{p q}}{3 E_{p q}^{3}}+b_{p} \frac{E_{p}^{2}}{E_{p q}^{4}}\right)
$$

with

$$
b_{p}=\frac{1.4 \ln p-0.7}{p}-\frac{0.51}{p^{2}}+\frac{1.16}{p^{3}}-\frac{0.55}{p^{4}}
$$

The value $\mathfrak{R}$ is the Rydberg constant and $k T_{e}$ is in $\mathrm{eV}, f_{p q}$ is the absorption oscillator strength given by Johnson (1972). For comparison SH use data from Percival \& Richards (1978). Again, differences can easily be a factor of 2 and are a basic problem (Chang, Avrett, \& Loeser 1991).

Case B does not define the populations of the $n=1$ or $n=2$ levels, so collisions from these levels are not included in the comparisons made below.

\subsection{Radiative Cascade}

This is the most difficult process to simulate, and the only one whose treatment is nonstandard, because the branching ratios from each level depend on both density and temperature. Physically, captures tend to occur to higher angular momentum, $l$, levels. As electrons cascade downward they further tend to "bunch up" at the highest possible $l$-values because of the $\Delta l=1$ selection rule. Distant collisions with slow-moving protons tend to distribute the electron density population according to statistical weight. As a result, the actual distribution of populations among the $l$-levels will have a density dependence, and the branching ratios from a particular level will too.

The dashed line in Figure 2 shows the $\mathrm{Pa} \alpha$ to $\mathrm{H} \beta$ ratio from $\mathrm{SH}$ for three temperatures. The lines have a common upper level, and so the intensity ratio is proportional to the 4-3/4-2 branching ratio. The lines are observationally important since both are strong and can be readily detected, and the ratio has a large dependency on the density and temperature of the gas. There are two simple limits; the well l-mixed, high-density limit with $\mathrm{Pa} \alpha / \mathrm{H} \beta=0.277$ (at all temperatures) corresponding to the Seaton (1959) recombination spectrum, and the low-density limit with $\mathrm{Pa} \alpha /$ $\mathrm{H} \beta=0.339$ (at $10,000 \mathrm{~K}$ ), where $l$-mixing collisions are negligible (corresponding to the calculations presented by Pengelly 1964 and Martin 1988).

We defined a transition probability from each excited level in terms of a constant total case B lifetime (the sum of the Einstein $A$ 's for the upper level minus the ground-state transition) and a set of branching ratios which depend on density and temperature, as the solid line in Figure 2 shows. These branching ratios were obtained by fitting the SH

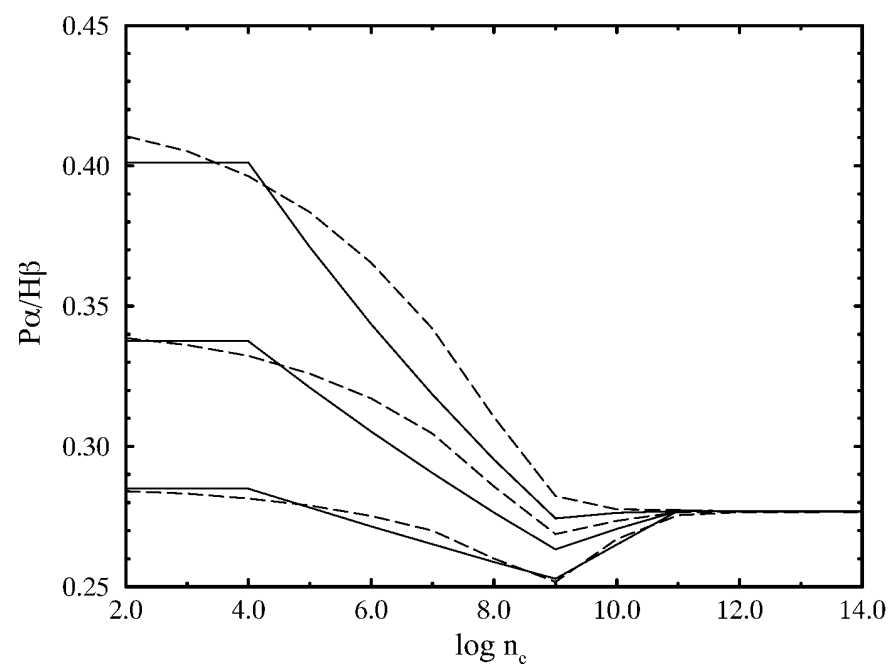

FIG. 2.- $\mathrm{SH}$ (dashed lines) predicted $\mathrm{Pa} \alpha$ to $\mathrm{H} \beta$ ratio as a function of density and temperature. Three temperatures are shown, $5000 \mathrm{~K}, 10,000 \mathrm{~K}$, and $20,000 \mathrm{~K}$, moving from top to bottom. Our fit to the branching ratio is shown as a solid line. 
TABLE 2

Fits OF THE BRANCHING RATIOS

\begin{tabular}{|c|c|c|c|c|c|c|c|}
\hline $\begin{array}{c}q \\
(1)\end{array}$ & $\begin{array}{c}p \\
(2)\end{array}$ & $\begin{array}{c}n_{\min } \\
(3)\end{array}$ & $\begin{array}{c}A \\
\text { (4) }\end{array}$ & $\begin{array}{c}B \\
(5)\end{array}$ & $\begin{array}{c}C \\
(6)\end{array}$ & $\begin{array}{c}D \\
\text { (7) }\end{array}$ & $\begin{array}{c}E \\
(8)\end{array}$ \\
\hline \multirow[t]{2}{*}{$4 \ldots \ldots$} & 2 & 9 & -0.1211 & 0.0603 & 0.4835 & 0.3618 & 0.0146 \\
\hline & 3 & & 1.1216 & -0.0604 & 0.5165 & 0.6382 & -0.0146 \\
\hline \multirow[t]{3}{*}{$5 \ldots \ldots$} & 2 & 8 & -0.2310 & 0.0555 & 0.3407 & 0.1284 & 0.0220 \\
\hline & 3 & & 0.1541 & 0.0136 & 0.2961 & 0.2895 & 0.0000 \\
\hline & 4 & & 1.0777 & -0.0692 & 0.3632 & 0.5808 & -0.0218 \\
\hline \multirow[t]{4}{*}{$6 \ldots .}$. & 2 & 8 & -0.2393 & 0.0490 & 0.2740 & 0.1240 & 0.0152 \\
\hline & 3 & & -0.0180 & 0.0226 & 0.2190 & 0.1822 & 0.0033 \\
\hline & 4 & & 0.2895 & -0.0061 & 0.2180 & 0.2562 & -0.0041 \\
\hline & 5 & & 0.9860 & -0.0668 & 0.2900 & 0.4378 & -0.0145 \\
\hline \multirow[t]{5}{*}{$7 \ldots \ldots$} & 2 & 7 & -0.2313 & 0.0441 & 0.2360 & -0.0102 & 0.0238 \\
\hline & 3 & & -0.0752 & 0.0244 & 0.1800 & 0.0889 & 0.0083 \\
\hline & 4 & & 0.1399 & 0.0024 & 0.1636 & 0.1619 & 0.0000 \\
\hline & 5 & & 0.3215 & -0.0135 & 0.1750 & 0.2684 & -0.0090 \\
\hline & 6 & & 0.8889 & -0.0615 & 0.2450 & 0.4915 & -0.0232 \\
\hline
\end{tabular}

results, for transitions from the first excited state up to $n=15$. Above $n=15$, the branching ratios were left at their high-density values given by the Einstein $A$ 's.

Branching ratios from the upper level $n \leq 7$ were calculated using two linear interpolations in temperature, one at $\log n_{e} \leq 4$ and one at $n_{e}=n_{\min }$, where $n_{\min }$ is defined as the minimum density of the branching ratio. For $n=4, \log$ $n_{\min }=9$ (see Fig. 2). At $\log n_{e} \geq 11$ (for transitions from the upper level $n=4$ ), the branching ratio is constant for all temperatures since the atom has gone over to the well $l$-mixed limit (see Fig. 2). For intermediate densities between the three points, $\log n_{e}=4, \log n_{e}=n_{\min }$, and $\log n_{e}=11$, we linearly interpolate the branching ratio in density.

Table 2 gives the coefficients of the fits used to calculate the branching ratios up to, and including, quantum level $n=7$. For $\log n_{e} \leq 4$, the branching ratio can be fitted with the equation $A+B \ln T_{e}$, and at $\log n_{e}=n_{\min }$ in column (3) of the table the branching ratio can be approximated with $D+E \ln T_{e}$. The value $C$ in column (6) in the table is the high-density branching ratio, good for $\log n_{e} \geq n_{\min }+2$. The temperature range for all fits of the branching ratio is $1000-30,000 \mathrm{~K}$, the valid range of $\mathrm{SH}$.

Table 3 gives the coefficients of the fits used to calculate the branching ratios for the atomic levels $n \geq 8$. For these levels the branching ratios were assumed to be simpler than Table 2 (since they are more nearly constant), such that the fits only interpolate in temperature for $\log n_{e} \leq 4\left(n_{\min }\right.$ is not defined). The upper level is denoted in column (1) of the table, and the transitions are listed horizontally to the right. The notation is the same as in Table 2: at $\log n_{e} \leq 4$, the branching ratio is $A+B \ln T_{e}$, and for $\log n_{e} \geq 11$, the branching ratio is $C$. For intermediate densities we linearly interpolate in density to calculate the branching ratio.

Errors in the branching ratios at high densities are generally less than $1 \%$, typically less than $0.5 \%$ since the branching ratios go to the high-density limit for all temperatures. Errors at low density are larger, on the order of $1 \%-2 \%$. The intermediate density range, $4 \leq \log n_{e} \leq 9$, errors are still larger, typically $5 \%-10 \%$, because of deviation from linearity of the branching ratios for highly excited levels. In what follows these errors will be small compared with other uncertainties.

\section{RESULTS}

The atom has been incorporated into the radiative equilibrium code CLOUDY (Ferland 1996). We have left the total number of levels of the hydrogen atom as an option. More levels generally produce a better agreement with the $\mathrm{SH}$ results, but at the expense of longer execution times. Tests show that lower temperatures require more levels, because the highest level must be well within $k T_{e}$ of the continuum for three-body recombination to achieve its full efficiency. A flexible choice in the number of levels was a major reason for the chosen structure of the model atom. For all of the results shown here a 50-level atom was used.

Figure $3 a$ shows a contour plot of the emissivity of $\mathrm{H} \beta$ (in units of $4 \pi j_{v} / n_{e} n_{p}$ ) for temperatures greater than $10^{3} \mathrm{~K}$ and the full range of densities considered by SH. The $\mathrm{H} \beta$ emissivity varies by 5 orders of magnitude for this range in conditions. Figure $3 b$ shows a ratio of this work and that of SH. For nebular temperatures $(5000-20,000 \mathrm{~K})$ and all densities, the differences are less than $2 \%$. For low temperatures $(<3000 \mathrm{~K})$ and low densities $\left(<10^{7} \mathrm{~cm}^{-3}\right)$, the general agreement is to within $6 \%$. In this limit the differences are due to our use of well $l$-mixed Einstein $A$ 's for the higher levels (see $\S 2.4$ ). At low temperatures, captures are mainly to these levels, which are not actually mixed at low densities. This is a basic limitation of our approach.

Uncertainties in the collision data (see $\S 2.2$ ) are the main reason our atom does not agree exactly with $\mathrm{SH}$ at low temperatures $(<3000 \mathrm{~K})$ and high densities $\left(>10^{8} \mathrm{~cm}^{-3}\right)$. We quantify the basic uncertainties by modifying the collisional rate coefficients from Vriens \& Smeets by a factor of 2 , the representative uncertainty discussed by Chang, Avrett, \& Loeser (1991). Figure $3 c$ shows the ratio of our predictions with and without this scale factor and so shows effects of these uncertainties. Results changed by nearly a factor of 2 at low temperatures and high densities, in response. We attribute the differences in our results and $\mathrm{SH}$ at low temperature and high densities to the fact that we use different sources for the collision data. The results for nebular conditions (5000-20,000 K) are affected very little, and we agree with $\mathrm{SH}$ very well.

Figure 4 shows our results with $\mathrm{SH}$ for four Balmer lines at $T_{e}=10^{4} \mathrm{~K}$ relative to $\mathrm{H} \beta$. At $\log n_{e}=2.0$ we calculate the Balmer decrement, $\mathrm{H} \alpha / \mathrm{H} \beta / \mathrm{H} \gamma / \mathrm{H} \delta$, to be $2.866 / 1.0 /$ $0.473 / 0.262$, and at $\log n_{e}=14.0$, it is $3.548 / 1.0 / 0.388 / 0.189$. The SH results for the same densities are 2.86/1.0/0.468/ 0.259 and $3.41 / 1.0 / 0.387 / 0.188$, respectively.

Figure 5 shows the intensities of two observationally important infrared emission lines at $T_{e}=10^{4} \mathrm{~K}$ relative to $\mathrm{H} \beta$. They agree with SH to typically within $1 \%-2 \%$, with 


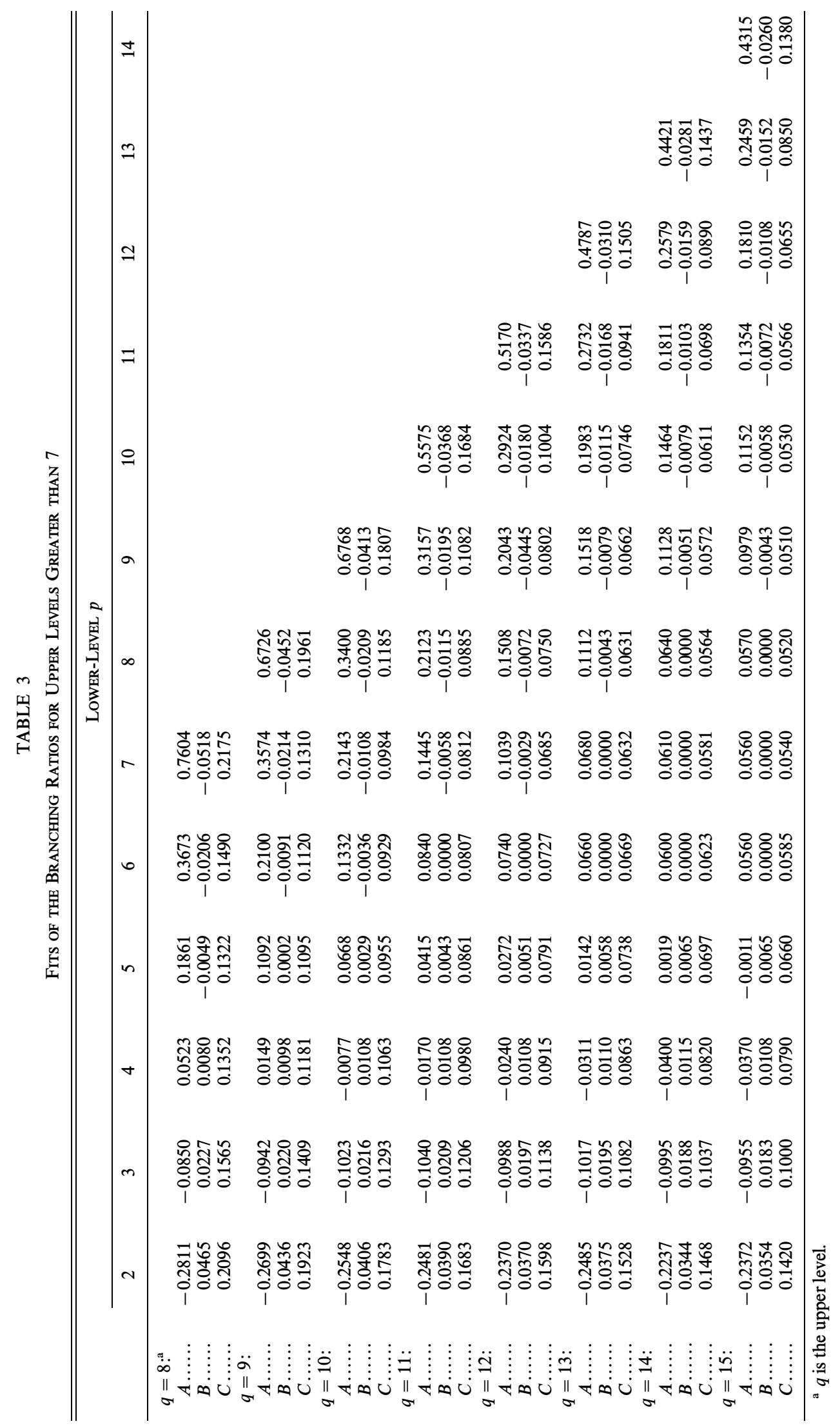




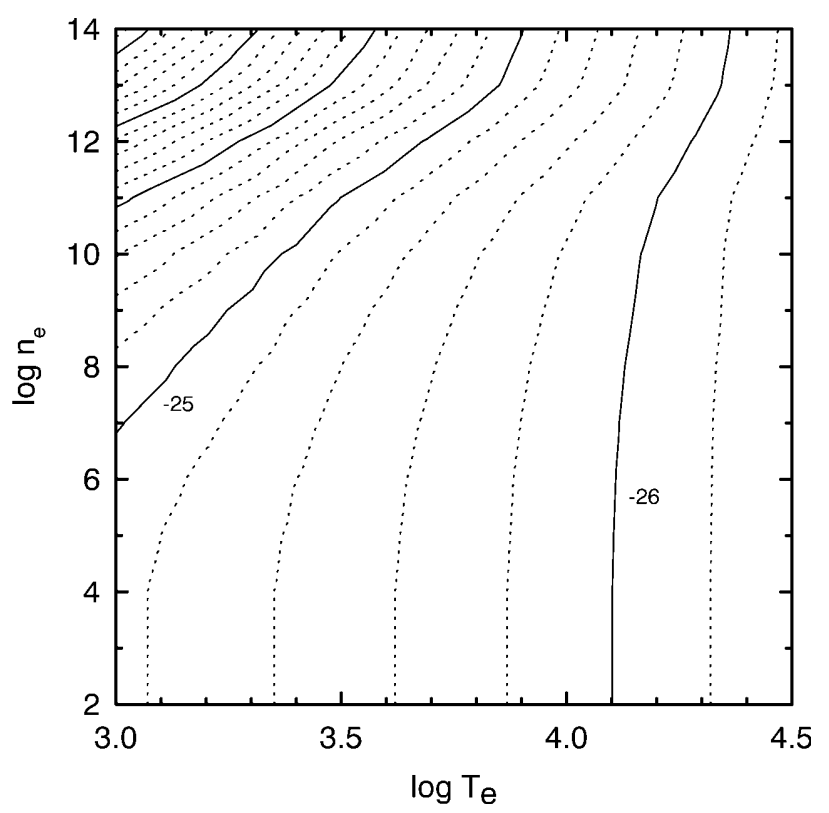

FIG. $3 a$

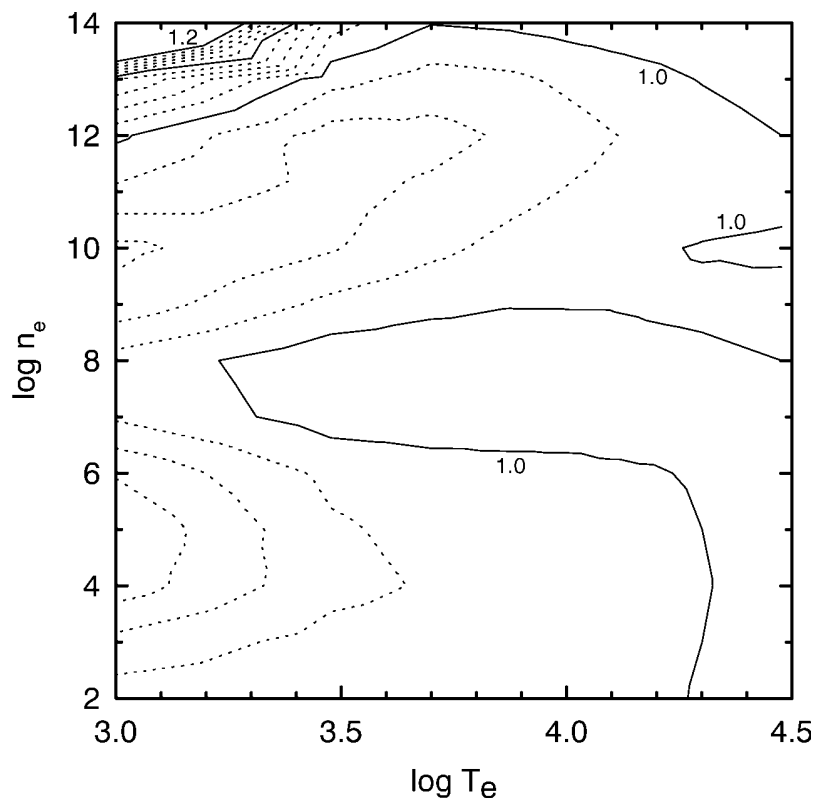

FIG. $3 b$

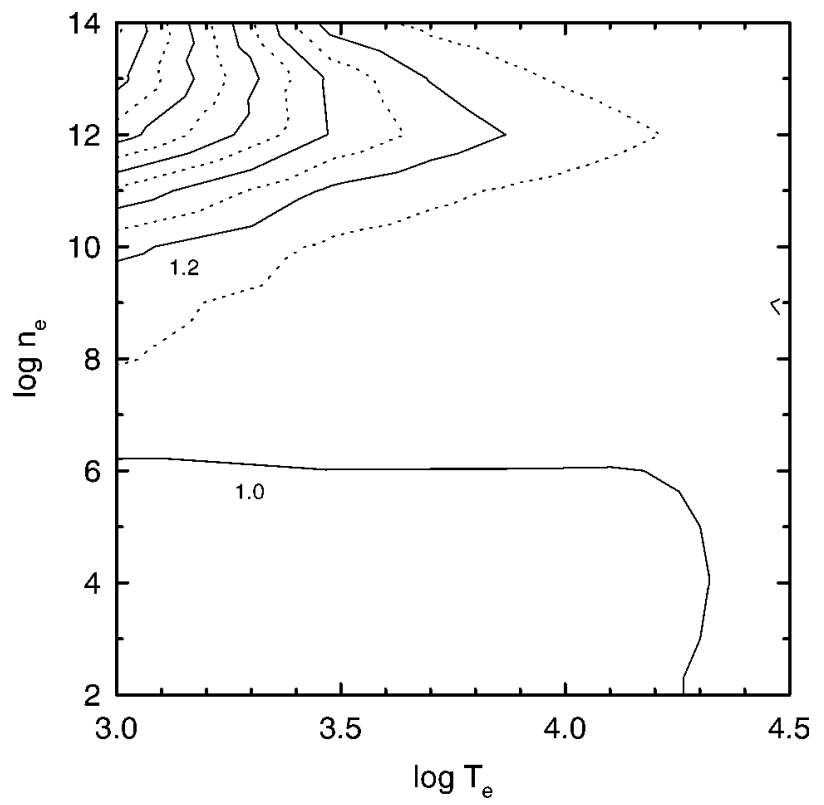

FIG. $3 c$

Fig. 3.-Contour plots. (a) The log of the total $\mathrm{H} \beta$ emissivity (in units of $4 \pi j_{v} / n_{e} n_{p}$ ) for all densities and temperatures. Solid lines are 1 dex increments and dashed lines are 0.2 dex steps. (b) Ratio of our predicted $\mathrm{H} \beta$ emissivity to $\mathrm{SH} ; 10 \%$ increments are solid lines, and $2 \%$ steps are dashed lines. $(c)$ Same as $(b)$, but with the collisional atomic data multiplied by 2 , as described in the text. In $(c)$ the solid lines are $20 \%$ differences and the dashed lines $10 \%$. Factor of 2 changes result for some parameters.

the largest errors being $5 \%$. Tests show that this accuracy is typical for lines produced by levels lower than 15 , the highest level we attempted to take account of variable $l$ mixing.

The case B approximation does not include the effects of the ground and the first excited states by design, so these results cannot be directly applied to dense clouds. Among the processes which were disabled for the comparisons above were continuum pumping, photoionization and induced recombination from excited states, and collisional excitation from the ground or first excited states. For dense clouds such as the BLR of a quasar, these are very important processes.
Table 4 compares case $B$ with this work for $10^{4} \mathrm{~K}$ and an electron density of $10^{11} \mathrm{~cm}^{-3}$, typical BLR conditions from Ferland et al. (1992). Column (1) lists the line labels, column (2) contains the case $\mathrm{B}$ ratios $(\mathrm{SH})$ with respect to $\mathrm{H} \beta$, and column (3) contains results from our compact atom with all induced processes disabled. Columns (4) and (5) show the effects of multiplying the collision data for levels higher than two by factors of 2 and 0.5 , respectively. Clearly the results are sensitive to these uncertain numbers. Column (6) shows the results of enabling induced photoionization and recombination and stimulated emission for a continuum shape and an ionization parameter (ratio of photon density to hydrogen density) of 0.1 , given by the standard BLR 

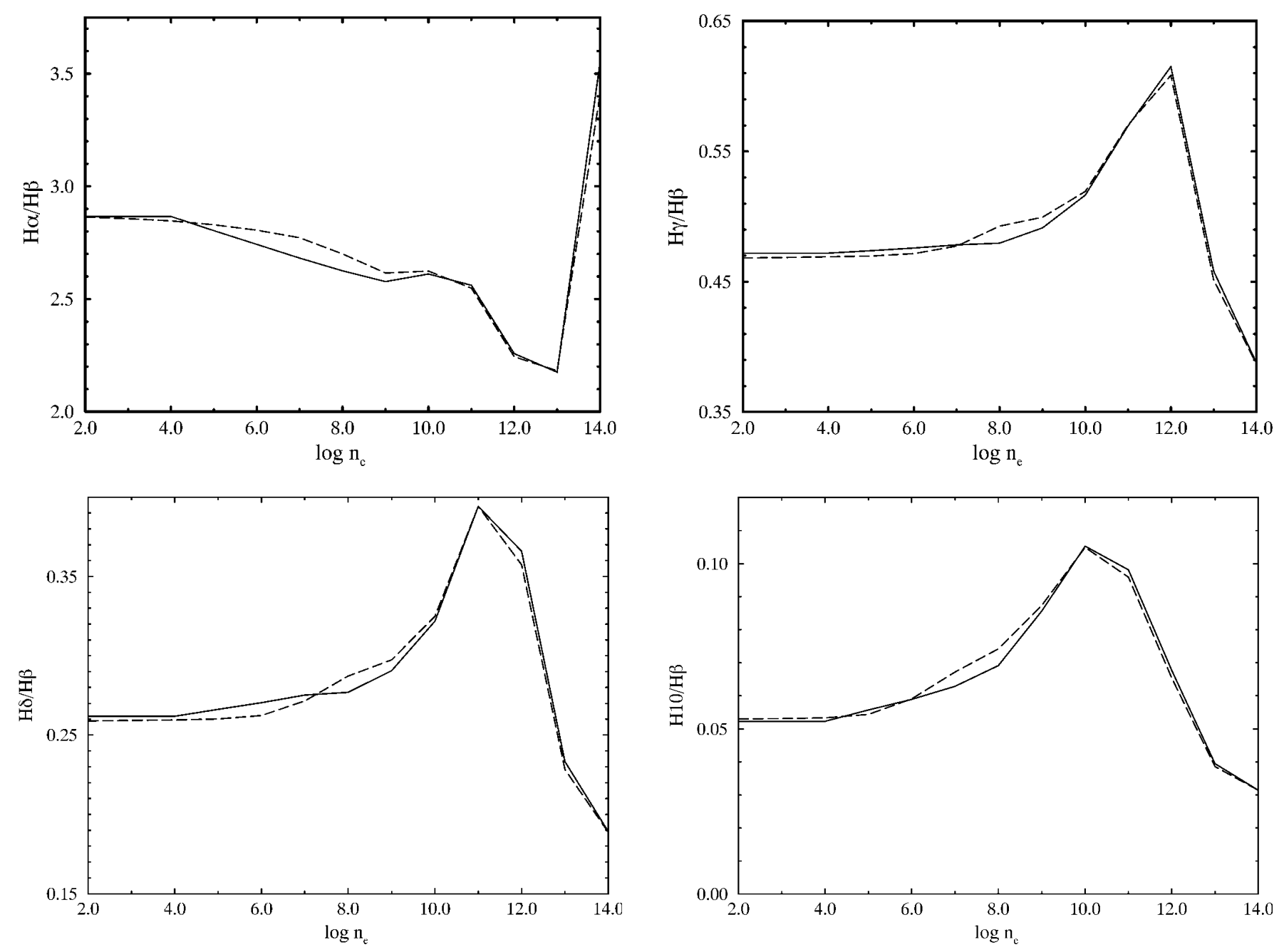

FIG. 4.-Selected ratios of Balmer lines relative to $\mathrm{H} \beta$ for $10^{4} \mathrm{~K}$ and the full range of density. This work is shown as solid lines and the SH results as dashed lines.

model from Ferland et al. (1992). Column (7) has collisions from the ground and first excited states included in the calculation. Column (8) lists the results of allowing the Lyman lines to be optically thin.

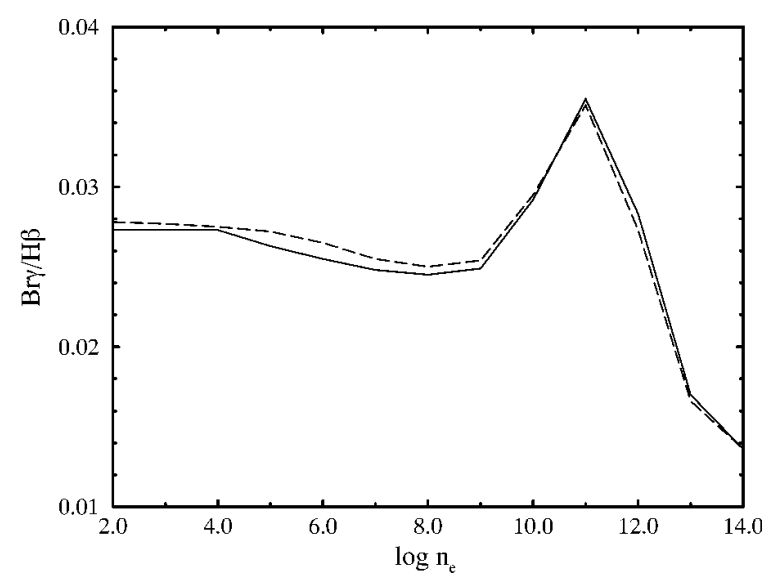

FIG. $5 a$
Finally, the results of a complete calculation are shown in column (9). This solves for the energy balance and so has a depth-dependent temperature (the mean is close to $10^{4} \mathrm{~K}$ ). It again assumes a hydrogen density of $10^{11} \mathrm{~cm}^{-3}$, has a

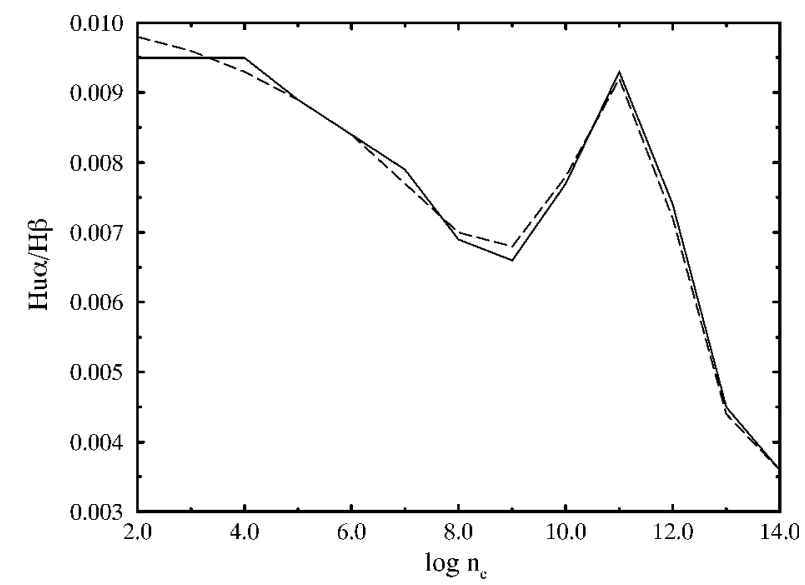

FIG. $5 b$

Fig. 5.-Two examples of important infrared lines shown at $10^{4} \mathrm{~K}$ and the full range of density. Solid lines: this work, dashed lines: $\mathrm{SH}$. (a) Br $\gamma$ to $\mathrm{H} \beta$; (b) $\mathrm{Hu} \alpha$ to $\mathrm{H} \beta$. Hu $\alpha$ is Humphries $\alpha$, the transition from level 7 to level 6 in hydrogen. 
TABLE 4

Case B Comparison with Model Atom

\begin{tabular}{|c|c|c|c|c|c|c|c|c|}
\hline \multirow[b]{2}{*}{$\begin{array}{l}\text { LINE } \\
(1)\end{array}$} & \multirow[b]{2}{*}{$\begin{array}{c}\text { CASE } B^{a} \\
\text { (2) }\end{array}$} & \multicolumn{6}{|c|}{ MODEL } & \multirow[b]{2}{*}{$\begin{array}{l}\mathrm{BLR}^{\mathrm{h}} \\
(9)\end{array}$} \\
\hline & & $\begin{array}{l}1^{\mathrm{b}} \\
(3)\end{array}$ & $\begin{array}{l}2^{c} \\
(4)\end{array}$ & $\begin{array}{l}3^{\mathrm{d}} \\
(5)\end{array}$ & $\begin{array}{l}4^{e} \\
(6)\end{array}$ & $\begin{array}{l}5^{f} \\
(7)\end{array}$ & $\begin{array}{l}6^{g} \\
(8)\end{array}$ & \\
\hline $\mathrm{H} \beta^{\mathrm{i}}$ & $1.84(-25)$ & $1.80(-25)$ & $1.95(-25)$ & $1.64(-25)$ & $2.94(-25)$ & $3.03(-25)$ & $1.25(-25)$ & $1.04(-27)$ \\
\hline $\operatorname{Ly} \alpha \ldots \ldots$ & 31.5 & & & & & & 34.68 & 65.6 \\
\hline $\mathrm{H} \alpha \ldots \ldots$ & 2.55 & 2.56 & 2.51 & 2.61 & 6.71 & 7.11 & 2.19 & 3.28 \\
\hline $\mathrm{H} \gamma \ldots \ldots$ & 0.570 & 0.570 & 0.594 & 0.545 & 0.4511 & 0.443 & 0.630 & 0.392 \\
\hline $\mathrm{H} \delta \ldots \ldots$ & 0.394 & 0.395 & 0.406 & 0.363 & 0.268 & 0.262 & 0.480 & 0.211 \\
\hline $\mathrm{H} 10 \ldots . .$. & 0.0959 & 0.0985 & 0.0906 & 0.101 & 0.0596 & 0.0578 & 0.138 & 0.104 \\
\hline $\mathrm{Pa} \alpha \ldots \ldots$ & 0.277 & 0.277 & 0.277 & 0.277 & 0.277 & 0.277 & 0.277 & 0.205 \\
\hline $\operatorname{Pa} \beta \ldots \ldots$ & 0.168 & 0.169 & 0.175 & 0.160 & 0.133 & 0.130 & 0.185 & 0.140 \\
\hline $\operatorname{Br} \alpha \ldots \ldots$ & 0.0654 & 0.0651 & 0.0679 & 0.0623 & 0.0515 & 0.0506 & 0.0719 & 0.0301 \\
\hline $\operatorname{Br} \gamma \ldots \ldots$ & 0.0351 & 0.0356 & 0.0347 & 0.0336 & 0.0220 & 0.0214 & 0.0465 & 0.0301 \\
\hline $\operatorname{Hu} \alpha \ldots \ldots$ & 0.0092 & 0.0093 & 0.0091 & 0.0088 & 0.0058 & 0.0056 & 0.0122 & 0.0022 \\
\hline
\end{tabular}

${ }^{a}$ Case $\mathrm{B}$ results are from $\mathrm{SH}$.

${ }^{\mathrm{b}}$ CLOUDY with physical processes disabled as described in text.

${ }^{c}$ Same as col. (3), but with collision data times 2 .

${ }^{\mathrm{d}}$ Same as col. (3), but with collision data times 0.5 .

e Induced processes enabled.

${ }^{f}$ Collisions between $2 s$ and $2 p$ electrons included.

${ }^{\mathrm{g}}$ Case B assumption turned off.

${ }^{\mathrm{h}}$ Simple BLR cloud as described in the text.

${ }^{\mathrm{i}} \mathrm{H} \beta$ emissivity in units of ergs $\mathrm{cm}^{3} \mathrm{sec}^{-1}$; number in parentheses is the exponent.

finite column density of $10^{26} \mathrm{~cm}^{-2}$ and corresponding $\mathrm{H}$-line optical depths, and has a nonthermal active galactic nucleus continuum. In the last case most hydrogen lines are optically thick, and both photoionization and collisional ionization from excited levels are very important. Clearly the line spectrum is far from case B. This underscores the influence of the ground and first excited state in highdensity situations.

In summary, we find that a compact hydrogen atom can reproduce quite well the hydrogen emission spectrum calculated with a more extensive model atom at most tem- peratures and densities. Our results underscore the points made by Chang, Avrett, and Loeser (1991), indicating the need for more accurate collisional rate coefficients.

The authors wish to thank K. T. Korista and D. A. Verner for very insightful discussion and questions throughout this work. We also thank the referee, G. Shields, for his insightful comments on our manuscript. Research in nebular astrophysics at the University of Kentucky is supported by the NSF through grant AST 93-19034 and by NASA with award NAG 5-3223.

\section{REFERENCES}

Abramowitz, M., \& Stegun, I. A. 1965, Handbook of Mathematical Functions (New York: Dover)

Allen, C. W. 1973, Astrophysical Quantities (London: Athlone)

Arnaud, M., \& Rothenflug, R. 1985, A\&AS, 60, 425

Avrett, E. H., \& Loeser, R. 1988, ApJ, 331, 211

Binette, L., Wang, J., Villar-Martín, M., Martin, P. G., \& Magris, C. M. 1993, ApJ, 414, 535

Brocklehurst, M. 1971, MNRAS, 153, 471

Burgess, A., \& Percival, I. C. 1968, Adv. At. Mol. Phys., 4, 109

Callaway, J. 1994, At. Data Nucl. Data Tables, 57, 9

Chang, E. S., Avrett, E. H., \& Loeser, R. 1991, A\&A, 247, 580

Drake, S. A., \& Ulrich, R. K. 1980, ApJS, 42, 351

Ferland, G. J. 1996, Hazy, a Brief Introduction to CLOUDY, Univ. Kentucky Dept. Phys. Astron. Internal Rep.

Ferland, G., et al. 1995, in STScI Symp. Ser., The Analysis of Emission Lines, ed. R. Williams \& M. Livio (Cambridge: Cambridge Univ. Press)

Ferland, G. J., Peterson, B. M., Horne, K., Welsh, W. F., \& Nahar, S. N. 1992, ApJ, 387, 95
Johnson, L. C. 1972, ApJ, 174, 227

Kallman, T., \& Mushotzky, R. 1985, ApJ, 292, 49

Martin, P. G. 1988, ApJS, 66, 125

Mathews, W. G., Blumenthal, G. R., \& Grandi, S. A. 1980, ApJ, 235, 971

Netzer, H. 1990, in Active Galactic Nuclei, ed. T. J.-L. Courvoisier \& M. Mayor (Berlin: Springer)

. 1996, in preparation

Osterbrock, D. 1989, Astrophysics of Gaseous Nebulae and Active Galactic Nuclei (Mill Valley: University Science Books)

Pagel, B. E. J. 1992, in Elements and the Cosmos, ed. M. Edmunds \& R Terlevich (Cambridge: Cambridge Univ. Press), 316

Pengelly, R. M. 1964, MNRAS, 127, 145

Percival, I. C., \& Richards, D. 1978, MNRAS, 183, 329

Rees, M. J., Netzer, H., \& Ferland, G. 1989, ApJ, 347, 640

Seaton, M. J. 1959, MNRAS, 119, 90

Shields, G. 1990, ARA\&A, 525

Storey, P. J., \& Hummer, D. G. 1995, MNRAS, 272, 41 (SH)

Vriens, L., \& Smeets, H. M. 1980, Phys. Rev. A, 22, 940 